\title{
Magnetic resonance markers of ischaemia: their correlation with vasodilatory reserve in patients with carotid artery stenosis and occlusion
}

\author{
D Lythgoe, A Simmons, A Pereira, M Cullinane, S Williams, H S Markus
}

Clinical Neuroscience, St George's Hospital Medical School, Cranmer Terrace, London, SW17 ORE, UK

A Simmons

A Pereira

M Cullinane

H S Markus

Neuroimaging Guy's, King's, and St Thomas' School of

Medicine and the

Institute of Psychiatry

D Lythgoe

A Simmons

$S$ Williams

Correspondence to :

Professor H Markus, Clinical

Neuroscience

h.markus@sghms.ac.uk

Received 21 August 2000 and in revised form

12 February 2001

Accepted 16 February 2001

\begin{abstract}
Objectives-Better methods of identifying patients with asymptomatic carotid artery stenosis who are at high risk of stroke are required. It has been suggested that proton magnetic resonance spectroscopy (MRS) may allow the identification of ongoing ischaemia in this patient group by the detection of a potentially reversible reduction of $\mathrm{N}$-acetyl aspartate (NAA), a presumed marker of neuronal integrity, and the presence of lactate, a marker of anaerobic metabolism. Previous studies have reported metabolite ratios rather than absolute concentrations. This study was performed to determine if NAA was reduced ipsilateral to carotid stenosis or occlusion, and if its concentration was related to carbon dioxide reactivity, a marker of cerebrovascular reserve.

Methods- Twenty one patients with unilateral carotid stenosis $(>70 \%)$ or occlusion were studied. Single voxel proton MRS was performed in the ipsilateral and contralateral hemispheres, with the voxel positioned in the arterial borderzone region between the middle and anterior cerebral artery territories. Absolute quantification of metabolite concentrations was performed. Cerebrovascular reactivity to $6 \%$ carbon dioxide was determined in both middle cerebral artery territories using transcranial Doppler ultrasonography.
\end{abstract}

Results-Mean (SD) cerebrovascular reactivity was significantly lower in the stenosed compared with the contralateral hemisphere (13.3 (7.7) $v 19.2(8.2) \% / k P a$, $p=0.002)$. There were no significant differences in the absolute concentrations of NAA, choline, or creatine between the ipsilateral and contralateral hemispheres (for example, NAA 10.1 (1.1) v 10.5 (1.1) mmol/1, $p=0.1)$. No lactate peak was seen in any spectra. For each metabolite measured, there was no correlation between the absolute concentration and cerebrovascular reactivity for either hemisphere.

Conclusions-In patients with carotid stenosis and occlusion we found no evidence that chronic hypoperfusion is associated with a reduction in NAA or the presence of lactate. Magnetic resonance spectroscopy is unlikely to help in the selection of patients with asymptomatic carotid stenosis for endarterectomy.

(F Neurol Neurosurg Psychiatry 2001;71:58-62)
Keywords: cerebrovascular disorders; spectroscopy; nuclear magnetic resonance; carotid artery diseases

Carotid endarterectomy reduces stroke risk in recently symptomatic patients with tight internal carotid artery stenosis. However, operating both on patients who had symptoms some time ago, and patients with asymptomatic carotid stenosis, seems to have limited benefit. ${ }^{1}$ Better methods are required to identify those patients with asymptomatic, or not recently symptomatic, carotid stenosis who are at high risk of stroke.

Magnetic resonance spectroscopy (MRS) has been proposed as a method to identify subclinical ischaemia in patients with carotid artery disease. N-Acetyl aspartate (NAA) concentration in the brain, estimated using proton MRS, is thought to be a marker of neuronal integrity. Decreased NAA occurs in various neurological disorders, such as cerebral infarction, ${ }^{2}$ and is usually interpreted as indicating irreversible neuronal damage. However, recent studies have shown recovery of NAA in some conditions, suggesting that reversible metabolic dysfunction may also result in reduced NAA concentration. ${ }^{3}{ }^{4}$ Therefore, cerebral ischaemia, in the absence of frank infarction, might result in a transient or permanent reduction of NAA. A series of studies from the same group have reported that NAA to choline (Cho), and NAA to creatine $(\mathrm{Cr})$ ratios are reduced in non-infarcted regions of the middle cerebral artery (MCA) territory ipsilateral to a stenotic carotid artery. ${ }^{5-10}$ As these studies measured metabolite ratios, such decreases could be caused by a decrease in NAA, or an increase in Cho or Cr. Absolute values of these metabolites would be of greater value than metabolite ratios. These studies also reported detection of lactate, a marker of anaerobic metabolism, which disappeared after carotid endarterectomy. ${ }^{6}$

If cerebral hypoperfusion in patients with carotid stenosis results in reduced NAA, it might be expected that this reduction would be seen to a greater extent in patients in whom intracerebral haemodynamics are most impaired. A proportion of patients with tight carotid stenosis have impaired cerebral haemodynamics, as determined by an increased oxygen extraction fraction ${ }^{11}$ or reduced vasomotor reactivity. ${ }^{12}$ Vasomotor reactivity is simply estimated by determining the increase in MCA blood flow velocity in response to the vasodilator carbon dioxide $\left(\mathrm{CO}_{2}\right)$. Patients with carotid occlusion who have reduced vasomotor reactiv- 
ity or cerebrovascular reserve are at increased risk of stroke. ${ }^{13}$ A correlation between $\mathrm{CO}_{2}$ reactivity and $\mathrm{NAA} /$ choline ratio has been reported in patients with carotid stenosis. ${ }^{10}$

In this study we determined absolute NAA concentrations in the non-infarcted borderzones between anterior and MCA territories in patients with unilateral carotid stenosis or occlusion. We correlated these values with MCA vasomotor reactivity.

\section{Methods}

PATIENT GROUPS

Twenty one patients (18 men), mean (SD) age 67 (9) years, with $\geqslant 70 \%$ unilateral carotid stenosis or occlusion were studied. The degree of stenosis, determined using carotid duplex, was $70 \%-89 \%$ in five, $90-99 \%$ in 11 , and occlusion in five patients. Twelve patients were asymptomatic with no symptoms for at least 2 years. Nine were symptomatic (six transient ischaemic attacks and three strokes) but in these mean (SD) time since an event was long at 205 (162) days. Five had infarcts on MR imaging in the ipsilateral hemisphere; in four of these they were in potential arterial borderzone regions, in two patients in the parieto-occipital borderzone between the middle and posterior cerebral artery territories, and in two in the centrum semiovale internal borderzone. Patients gave informed written consent. The protocol was approved by the local hospital ethics committee.

\section{TRANSCRANIAL DOPPLER REACTIVITY}

MEASUREMENTS

Continuous simultaneous bilateral MCA insonation was performed using a commercially available TCD machine (Multi Dop x4, DWL Electronische $\mathrm{GMbH}$ ). A face mask was used to deliver air or a $\mathrm{CO}_{2}$ /air mixture, and sampling of end tidal (ET) $\mathrm{CO}_{2}$ (Datex Normocap 200). Once steady state was reached for both the ET $\mathrm{CO}_{2}$ and MCA flow velocities while breathing air, bilateral MCA flow velocities and ET $\mathrm{CO}_{2}$ were recorded for 30 seconds to obtain baseline readings. The $6 \% \mathrm{CO}_{2}$ /air mixture was then administered for 2-3 minutes, until a steady state was reached, when measurements were again made over a 30 second period. A reactivity index was calculated from the percentage rise in MCA velocity divided by the absolute rise in ET $\mathrm{CO}_{2}$ measured in $\mathrm{kPa}$. Studies with TCD were usually performed on the same day as the MR studies and in all cases within 2 weeks.

MAGNETIC RESONANCE IMAGING AND SPECTROSCOPY

A 1.5T GE Signa (GE Medical Systems) MR system with a quadrature transmit and receive high pass radiofrequency coil was used. An 11 slice SPGR sagittal localiser image (TR $=150$ $\mathrm{ms}, \mathrm{TE}=42 \mathrm{~ms}, \alpha=90^{\circ}, \Delta \mathrm{x}=\Delta \mathrm{y}=0.94 \mathrm{~m}$, field of view $(F O V)=240 \mathrm{~mm} \times 240 \mathrm{~mm}$, slice thickness $=5.0 \mathrm{~mm}$, slice gap $=2.5 \mathrm{~mm}$ ) was used to prescribe near axial dual echo fast spin echo images from the whole head $(T R=4000$ $\mathrm{ms}, \mathrm{TE} 1=17 \mathrm{~ms}, \mathrm{TE} 2=102 \mathrm{~ms}, \alpha=90^{\circ}$,
$\Delta \mathrm{x}=\Delta \mathrm{y}=0.86 \mathrm{~mm}, \mathrm{FOV}=220 \mathrm{~mm} \times 220 \mathrm{~mm}, 50$ slices with slice thickness $=3.0 \mathrm{~mm}$ ).

Single voxel proton spectroscopy was performed using an automated spectroscopy acquisition technique. $8 \mathrm{~cm}^{3}$ voxels $(2 \mathrm{~cm} \times 2 \mathrm{~cm} \times 2 \mathrm{~cm})$ were prescribed in the borderzone region between the MCA and anterior cerebral arteries with a grey to white matter ratio of about $60 \%$ to $40 \%$, in identical positions in both the ipsilateral and contralateral hemispheres. Care was taken to avoid infarcted tissue. In one of the patients, voxels were prescribed in the borderzone region between the MCA and posterior cerebral artery arterial territories, due to the presence of infarction in the anterior borderzone region.

For each voxel, proton MR spectra were obtained using a point resolved spectroscopy (PRESS) sequence with a TR of $2000 \mathrm{~ms}$, a TE of $45 \mathrm{~ms}$, and a spectral width of $2500 \mathrm{~Hz}$ from which 2000 data points were collected. Before spectra acquisition, an automated shimming procedure was used to optimise $\mathrm{B}_{0}$ homogeneity. For the metabolite spectra, 256 data averages were acquired, with an eight step phase cycle. Water suppression was performed using chemical shift selective (CHESS) RF pulses with a bandwidth of $75 \mathrm{~Hz}$ and associated spoiling gradients to excite and then dephase the water signal before application of the spatial localisation sequence. A flip angle of greater than $90^{\circ}$ was used for the final CHESS $\mathrm{RF}$ pulse to allow for $\mathrm{T} 1$ relaxation. The flip angle of this last pulse was automatically optimised to minimise the residual water signal. For each voxel, unsuppressed water spectra were also acquired with 32 averages.

ANALYSIS OF MAGNETIC RESONANCE SPECTRA Metabolite concentrations were calculated using the LC Model method which compares an in vivo spectrum with spectra derived from metabolite solutions in vitro. ${ }^{14}$ Resonance peaks were assigned with $\mathrm{Cr}$ at $3.03 \mathrm{ppm}$, Cho at $3.22 \mathrm{ppm}, \mathrm{NAA}$ at $2.01 \mathrm{ppm}$, and the lactate doublet at $1.33 \mathrm{ppm}$ with a $7 \mathrm{~Hz}$ splitting. The water resonance at $4.7 \mathrm{ppm}$ was used as the internal standard, assuming a concentration of 41.7 M. ${ }^{15}$ Metabolite concentrations were corrected for T1 and T2 relaxation assuming previously described metabolite relaxation rates. ${ }^{16}$ The coefficient of variation for NAA concentrations using this same system if $5 \%{ }^{17}$

Absolute concentrations of each of the metabolites were compared between the ipsilateral and contralateral hemispheres using Student's paired $t$ test. Relations between

Table 1 Metabolite concentrations in the hemispheres ipsilateral and contralateral to the carotid stenosis/occlusion

\begin{tabular}{llll}
\hline & \multicolumn{3}{l}{ Metabolite concentration (mmol/l) } \\
\cline { 2 - 4 } & $\begin{array}{l}\text { Ipsilateral } \\
\text { hemisphere }\end{array}$ & $\begin{array}{l}\text { Contralateral } \\
\text { hemisphere }\end{array}$ & p Value \\
\hline Choline & $1.38(0.28)$ & $1.40(0.31)$ & 0.708 \\
Creatine & $8.19(0.95)$ & $8.30(1.00)$ & 0.457 \\
N-Acetyl aspartate & $10.1(1.1)$ & $10.5(1.1)$ & 0.100 \\
\hline
\end{tabular}

Concentrations are expressed as mean (SD). The $p$ values are from the comparison between the two hemispheres using paired student's $t$ test. 

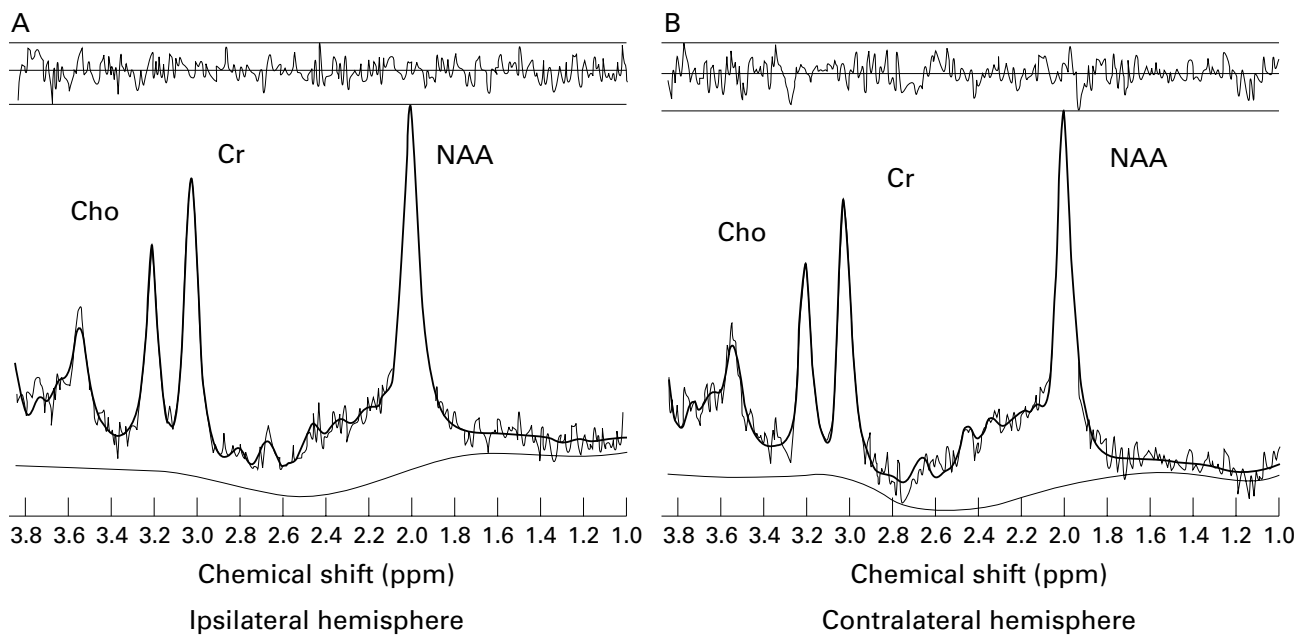

Figure 1 An example of a typical spectra and their analysis. On the left is the spectrum from the ipsilateral side and on the right the contralateral side. In the lower panels the original spectra are shown with the fitted curve from the LC model superimposed and shown in bold. In the upper panel are the residuals from the LS model fitting. The curve at the bottom is the baseline.

Table 2 Relation between absolute metabolite concentrations and transcranial Doppler cerebrovascular reactivity for both hemispheres combined, and the ipsilateral and contralateral hemispheres separately

\begin{tabular}{|c|c|c|c|c|c|c|}
\hline & \multicolumn{2}{|c|}{ Both hemispheres } & \multicolumn{2}{|c|}{ Ipsilateral hemisphere } & \multicolumn{2}{|c|}{ Contralateral hemisphere } \\
\hline & $r^{2}$ & $p$ Value & $r^{2}$ & $p$ Value & $r^{2}$ & $p$ Value \\
\hline Choline & $<0.001$ & 0.949 & 0.070 & 0.247 & 0.038 & 0.396 \\
\hline Creatine & 0.015 & 0.439 & $<0.001$ & 0.928 & 0.038 & 0.399 \\
\hline $\mathrm{N}$-Acetyl aspartate & 0.050 & 0.156 & 0.024 & 0.498 & 0.033 & 0.431 \\
\hline
\end{tabular}

metabolite concentrations and the normalised TCD reactivity were calculated individually for the ipsilateral and contralateral hemispheres, and for values from both hemispheres together, using Pearson's correlation coefficient.

\section{Results}

Mean (SD) normalised $\mathrm{CO}_{2}$ reactivity was lower in the stenosed hemisphere compared with the non-stenosed hemisphere (13.3 (7.7) v $19.2(8.2) \% / \mathrm{kPa}, \mathrm{p}=0.002)$. There were no significant differences in absolute concentrations of any of the metabolites between the two hemispheres (table 1). No lactate resonance was detectable on any spectra. Figure 1 shows typical spectra from the ipsilateral and contralateral hemispheres and the output from the LC model fitting.

For each metabolite there was no correlation between the absolute concentration and the normalised reactivity, either for both hemispheres combined, or for the ipsilateral or contralateral hemispheres alone. The correlation coefficients for the relations between reactivity and metabolites are shown in table 2; for all relations $r^{2}$ was near zero.

We also analysed relations using metabolite ratios. There was no difference in mean (SD) ratios between the ipsilateral and contralateral hemispheres: $1.27(0.17)$ v 1.26 (0.19), $\mathrm{p}=0.940$ for NAA/Cr and $6.74(1.24) v 6.77$ (1.57), $\mathrm{p}=0.918$ for NAA/Cho. There was no relation between NAA/Cho ratios and reactivity for the results from both hemispheres $\left(r^{2}\right.$ $=0.034, \mathrm{p}=0.245$ ) or the ipsilateral hemisphere only $\left(r^{2}=0.204, p=0.040\right)$. There was no relation between NAA/Cr ratios and reactivity for both hemispheres $\left(r^{2}=0.010, \mathrm{p}=0.530\right)$, or the ipsilateral hemisphere only $\quad\left(r^{2}=0.039\right.$, $\mathrm{p}=0.392)$.

\section{Discussion}

We found no evidence of reduced NAA or the presence of lactate in the MCA territory ipsilateral to a carotid stenosis or occlusion. This was despite the fact that we determined metabolite concentrations in the region of the arterial borderzones, although the position of these is difficult to precisely localise due to between patient variation in cerebral arterial territories. These borderzone territories are distal to the origin of the feeding arteries, and most vulnerable to a drop in cerebral perfusion pressure. By contrast, we confirmed the previous finding that cerebrovascular reserve in the ipsilateral hemisphere is reduced in some patients with carotid stenosis and occlusion. ${ }^{12} 13$ However, we found no relation between this haemodynamic measure and NAA. Therefore our study does not support the hypothesis that MRS allows identification of a high risk group of patients with carotid artery disease.

The finding of reduced NAA ipsilateral to an asymptomatic carotid stenosis does not necessarily imply that haemodynamic factors are responsible for the reduction. Recent studies have demonstrated that asymptomatic embolisation is common distal to both symptomatic and asymptomatic carotid stenosis. ${ }^{18}$ Such subclinical ischaemic insults could result in neuronal injury with both a reduction in NAA and the presence of lactate. Such changes might reverse after carotid endarterectomy; the incidence of asymptomatic embolisation falls 
markedly after the procedure. ${ }^{19}$ For this reason we not only looked for any difference in NAA concentrations between the two hemispheres, but we also correlated NAA concentrations with a measure of cerebral haemodynamics, $\mathrm{CO}_{2}$ reactivity. Impaired $\mathrm{CO}_{2}$ reactivity occurs in a subgroup of patients with carotid stenosis and occlusion. ${ }^{12}$

By contrast with our findings Van der Grond et al have reported a decreased NAA/Cho ratio in the MCA territory ipsilateral to carotid stenoses and occlusions. ${ }^{5-8}$ They have also reported the presence of lactate in patients with carotid stenosis. There are important differences between these studies and our current study, both in patient selection and data acquisition and analysis. The control group was younger and it is known that NAA decreases with age. Our study used the contralateral hemisphere as a control to eliminate bias due to poor case-control matching. All patients they studied had experienced a recent transient ischaemic attack and stroke (within 12 weeks), and many had regions of infarction in the stenosed carotid artery territory. In a significant proportion these were in the arterial borderzones. By contrast seven of our patients were asymptomatic and among our symptomatic patients the mean time from symptoms was long at 209 days. Only five had infarcts in the symptomatic hemisphere. Previous studies in acute stroke have shown the presence of lactate weeks after the acute event, even in regions of the brain appearing normal on MR imaging. ${ }^{2}$ Therefore the detection of lactate could be due to the short time period between symptoms and imaging. Similarly, the disappearance of lactate after carotid endarterectomy ${ }^{6}$ could merely reflect the natural history of increases in lactate after acute stroke. No unoperated control group was studied. We did not find lactate in any of our patients. Magnetic resonance spectroscopy is sensitive to small increases in lactate; concentrations as low as $0.3-0.90 \mathrm{mM}$ could be detected during photic stimulation. ${ }^{20}$

There were also technical differences between our study and those of Van der Grond et al. We placed a single voxel in one relatively small region of the hemisphere, whereas in the study of Van der Grond et al in which a single voxel was used this was a large voxel placed over most of the MCA. ${ }^{7}$ We chose a region in which haemodynamic compromise would be expected to be maximal-namely, the borderzone region. Van der Grond et al used different acquisition parameters with a long TE of 272 $\mathrm{ms},{ }^{59}$ by contrast with the short TE of $45 \mathrm{~ms}$ used by us. They measured metabolite ratios, whereas our study used absolute quantitation of metabolite concentrations. A fall in NAA/ Cho ratio could be caused by a fall in NAA, or a rise in choline, and therefore determination of absolute concentrations is important. Such absolute measurements, using tissue water as an internal standard, have been shown to produce reliable and reproducible results with the MR system used for this study. ${ }^{17}$ However, when we analysed NAA/Cho and NAA/Cr ratios we found no interhemispheric difference, and no relation with cerebrovascular reserve.
The use of single voxel spectroscopy may have introduced a sampling bias into our results as ischaemic brain regions may have been overlooked during placement of the voxel. A spectroscopic imaging technique might be less prone to this potential error. However, a single voxel technique was used in an attempt to determine absolute metabolite concentrations in the volumes of interest. Although it may be technically feasible to determine absolute metabolite concentrations in a spectroscopic imaging dataset, ${ }^{21}$ these methods are still not widely used, whereas robust methods are available to measure absolute metabolite concentrations in single voxel experiments. ${ }^{16}{ }^{17}$ Despite greater spatial coverage with multivoxel chemical shift imaging techniques, such techniques suffer from comprimised field homogeneity as sharp resonances are now required over a much greater extent of the brain. This leads to broader linewidths, poorer water suppression, and longer acquisition times. The need for long examination times and poorer signal to noise ratio makes the estimation of metabolite concentrations in a given volume of interest even more inaccurate..$^{22}$

Transcranial Doppler has poor spatial resolution, and estimates of reactivity are from the whole MCA territory. Its advantage is that studies have shown, in reasonable sample sizes, that poor reserve is associated with increased stroke risk. ${ }^{13}$ Using alternative methods which can measure regional perfusion with a higher spatial resolution, such as PET or SPECT, allows small areas of impaired reactivity to be identified. It is possible that if areas with maximal haemodynamic compromise had been identified using such techniques, and the MRS voxel placed in that region, that the chance of detecting areas of abnormal metabolism might have been increased.

Our findings are consistent with reduced perfusion pressure alone, in the absence of frank infarction, not resulting in a reduction in NAA. An alternative explanation is that the degree of reduction in perfusion pressure seen in these patients with carotid artery stenosis does not cause sufficient ischaemia to reduce NAA or raise lactate concentrations. Whether the perfusion pressure reduction secondary to a carotid artery stenosis does cause subtle, and possibly reversible, neurological or neuropsychological damage has been debated, with conflicting results published. ${ }^{23}$

In summary, we found no evidence that carotid artery stenosis results in a reduction of NAA, or the presence of lactate, in noninfarcted regions of the carotid artery territory. Magnetic resonance spectroscopy is unlikely to provide useful information in the selection of patients with asymptomatic carotid stenosis for endarterectomy.

This work was supported by a project grant from South Thames National Health Service Research and Development. DL was supported by the Stroke Association.

1 Chambers BR, You RX, Donnan GA. Carotid endarterectomy for asymptomatic carotid stenosis (Cochrane review). Oxford: for asymptomatic carotid stenosis (Cochrane review).

2 Saunders DE, Howe FA, Van den Boogaart A, et al. Continuing ischaemic damage after acute middle cerebral 
artery infarction in humans demonstrated by short-echo proton spectroscopy. Stroke 1995;26:1007-13.

3 De Stefano N, Mathews PM, Arnold DL. Reversible decreases in N-acetylaspartate after acute brain injury. Magn Reson Imaging 1995;34:721-7.

4 Narayama PA, Doyle TJ, Lai D, et al. Serial proton magnetic resonance spectroscopic imaging, contrast-enhanced magnetic resonance imaging, and quantitative lesion volumetry in multiple sclerosis Ann Neurol 1998;43:56-71.

5 van der Grond J, Balm R, et al. Cerebral metabolism of patients with stenosis or occlusion of the internal carotid artery. A ${ }^{1}$ H-MR spectroscopic imaging study. Stroke 1995 26:822-8

6 van der Grond J, Balm R, Klijn CJM, et al. Cerebral metabolism of patients with stenosis of the internal carotid artery before and after endarterectomy. 7 Cereb Blood Flow Metab 1996;16:320-6.

7 van der Grond J, Eikelboom BC, Mali WPTM. Flow-related anaerobic metabolic changes in patients with severe stenosis of the internal carotid artery. Stroke 1996;27:2026-32.

8 van der Grond J, Ramos LMP, Eikelboom BC, et al. Cerebral metabolic differences between the severe and Cerebral metabolic differences between the severe and

critical hypoperfused brain. Neurology 1996;47:399-404.
van der Grond J, van Everdingen KJ, Eikelboom BC, et al. Assessment of borderzone ischemia with a combined MR imaging-MR angiography-MR spectroscopy protocol. F Magn Reson Imaging 1999;9:1-9.

10 Visser GH, van der Grond J, van Huffelen AC, et al. Decreased transcranial Doppler carbon dioxide reactivity is associated with disordered cerebral metabolism in patients with internal carotid artery stenosis. F Vasc Surg 1999;30: 252-60.

11 Derdeyn CP, Yundt KD, Videen TO, et al. Increased oxygen extraction fraction is associated with prior ischemic events
in patients with carotid occlusion. Stroke 1998;29:754-8.

12 Ringlestein EB, Sievers C, Ecker S, et al. Non-invasive assessment of $\mathrm{CO}_{2}$-induced cerebral vasomotor response in normal individuals and patients with internal carotid artery clusions. Stroke 1988;19:963-9.

13 Kleiser B, Widder B. Course of carotid artery occlusions with impaired cerebrovascular reactivity. Stroke 1992;23: $171-4$.

14 Provencher SW. Estimation of metabolite concentrations from localized in vivo proton NMR spectra. Magn Reson Med 1993;30:672-9.

15 Norton WT, Poduslo SE, Suzuki K. Subacute sclerosing leukoencephalitis II: Chemical studies including abnormal myelin and abnormal ganglioside pattern. $\mathcal{f}$ Neuropathol Exp Neurol 1966;25:582-97.

16 Henriksen $\mathrm{O}$. In vivo quantitation of metabolite concentrations in the brain by means of proton MRS. NMR Biomed 1995;8:139-48

17 Simmons A, Smail M, Moore E, et al. Serial precision of metabolite peak areas in proton MR spectroscopy of the human brain. Magn Reson Imaging 1998;16:319-30.

18 Markus HS, Thomson N, Brown MM, et al. Asymptomatic cerebral embolic signals in symptomatic and asymptomatic carotid artery disease. Brain 1995;118:1005-11.

19 Siebler M, Sitzer M, Rose G, et al. Silent cerebral embolism caused by neurologically symptomatic high-grade carotid stenosis. Event rates before and after carotid endarterectomy. Brain 1993;116:1005-15.

20 Prichard J, Rothman D, Novotny E, et al. Lactate rise detected by ${ }^{1} \mathrm{H}$ NMR in human visual cortex during physiologic stimulation. Proc Natl Acad Sci USA 1991;88:5829-31.

21 Soher BJ, van Zijl PCM, Duyn JH, et al. Quantitative proton MR spectroscopic imaging of the human brain. Magn Reson Med 1996;35, 356-63.

22 Howe FA, Maxwell RJ, Saunders DE, et al. Proton spectroscopy in vivo. Magnetic Resonance Quarterly 1993;9:31-59.

23 Lunn S, Crawley F, Harrison $\mathrm{MJH}$, et al. Impact of carotid endarterectomy upon cognitive functioning. Cerebrovasc Dis 1999;9:74-81.

\section{Emil Adolph von Behring (1854-1917) and Shibasaburo Kitasato (1852-1931)}

Alhough many of his ideas are now obsolete, von Behring was one of the founders of immunology. He was the recipient of the first Nobel Prize in physiology or medicine awarded in 1901 and was elevated to the Prussian nobility in the same year.

After graduating in medicine from Berlin he entered the Army Medical Corps and in 1888 became a lecturer at the Army Medical College. He joined the Robert Koch Institute of Hygiene in 1889 and in 1891 the Institute of Infectious Diseases, when Koch was appointed its chief.

Von Behring experimented initially with iodine trichloride and zinc chloride as potential treatments for diphtheria and tetanus infections. In 1898, working with Koch's Japanese student Shibasaburo Kitasato, Behring showed that injections of serum from an animal with tetanus could confer immunity to the disease in other animals, and also that the same was true for diphtheria. In collaboration with Paul Ehrlich a diphtheria antitoxin for humans was developed and was first used on Christmas Eve in 1891. In the next year a dramatic fall in mortality from diphtheria occurred. The death rate from diphtheria in Berlin children's hospitals dropped from $48 \%$ to $13 \%$. In later life he worked to establish immunity against diphtheria in children by giving them a combination of diphtheria toxin and antitoxin. He also performed research on tuberculosis.

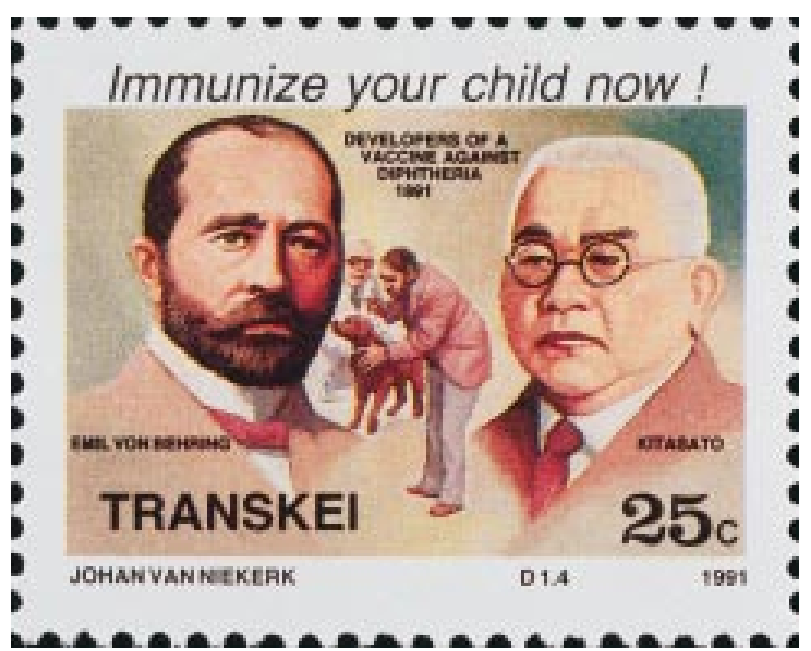

Emil von Behring and Shibasaburo Kitasato were honoured philatelically on a stamp issued by Transkei in 1991 (Stanley Gibbons 273, Scott 255). 